\section{Beta-Blocker, ACE-Hemmer und spezifische Immuntherapie}

\author{
Zum DGAI-Positionspapier „Die spezifische Immuntherapie (Hypo- \\ sensibilisierung) mit Allergenen“, Allergo Journal 2000; 9:317-24, \\ erreicht uns aus Wien folgender Kommentar mit Ergänzungen zur \\ besonderen Situation von Patienten, die Beta-Blocker oder ACE- \\ Hemmer einnehmen.
}

B ei unter Beta-Blocker- oder ACEHemmer-Therapie stehenden Patienten sind in den letzten Jahren im Zuge der Allergen-spezifischen Immuntherapie (SIT) wiederholt schwer verlaufende, anaphylaktische Reaktionen beschrieben worden. Auch im kürzlich erschienen Positionspapier zur spezifischen Immuntherapie der DGAI (1) wird explizit auf die mit diesen Arzneimitteln im Zusammenhang stehende Problematik hingewiesen und eine aktuelle Therapie mit solchen Präparaten unter den relativen Kontraindikationen für eine SIT aufgelistet.

Angesichts der ständig steigenden Zahl von Patienten unter dauernder Beta-Blocker- oder ACE-HemmerTherapie erscheint es aber bedauerlich, bei der betroffenen Patientengruppe hinsichtlich der Durchführung einer Hyposensibilisierung zu zurückhaltend zu sein oder sie aus Verunsicherung von dieser einzigen kausalen Therapie allergischer Erkrankungen kategorisch auszuschließen. Dies auch deshalb, weil die Problematik nicht so sehr in einem erhöhten Risiko systemischer Nebenwirkungen bei diesen Patienten besteht gerade dies wurde nämlich in der von den Autoren des Positionspapieres in Zusammenhang mit der relativen Kontraindikation bei Beta-Blocker- bzw. ACE-Hemmer-Therapie zitierten Arbeit (2) für die Beta-Blocker gezeigt, sondern vielmehr im potenziell schwierigeren Management allfälliger anaphylaktischer Reaktionen. Ist die Medikamentenanamnese bekannt, sind solche anaphylaktischen Reaktionen auch bei Patienten unter Beta-Blockern oder ACE-Hemmern gut beherrschbar.

Es erscheint uns daher sinnvoll, in Ergänzung zum zitierten Positions- papier der DGAI kurz auf die spezifische notfallmedizinische Situation bei Patienten unter Beta-Blocker- bzw. ACE-Hemmer-Therapie einzugehen:

$\checkmark$ Patienten mit Beta-Blocker- oder ACE-Hemmer-Therapie müssen durch sorgfältige Anamnese sicher erfasst werden, damit im Falle einer systemischen Reaktion eine adäquate Therapie eingeleitet werden kann.

- Wenn kein entsprechend geschultes Personal und keine ensprechende Notfallausrüstung vorhanden sind, sollte keine SIT durchgeführt werden.

- Eventuell auftretende systemische Nebenwirkungen müssen hier noch frühzeitiger und genauer erkannt werden.

- Bei Auftreten systemischer Symptomatik sollte der Patient aus Sicherheitsgründen frühzeitig einen intravenösen Zugang erhalten.

\section{Beta-Blocker-Therapie}

Patienten unter Beta-Blocker-Therapie benötigen auf Grund der Rezeptorenblockade u.U. eine höhere Dosis an Katecholaminen (Adrenalin, Noradrenalin). Dopamin ist wegen der sich überschneidenden, zwar dosisabhängigen dopaminergen, alpha- und beta-adrenergen Wirkungen nicht wirklich gut steuerbar und stark arrhythmogen wirksam und daher nicht empfehlenswert. Diese Patienten sind aber mit adäquater Volumensubstitution (Kristalloide oder Kolloide) erfolgreich zu behandeln.

\section{ACE-Hemmer-Therapie}

Patienten unter ACE-Hemmer reagieren wegen des herabgesetzten peripheren Gefäßwiderstands träge auf eine Volumensubstitution, sprechen aber auf
Katecholamine (Adrenalin, insbesondere Noradrenalin) gut an. Diese müssen dann rechtzeitig verabreicht werden.

\section{Fazit}

Zusammenfassend ist demnach auch bei den oben genannten Patienten eindeutig für die SIT zu entscheiden,

D weil schwere systemische Nebenwirkungen extrem selten sind und

- weil bei sorgfältiger Anamnese und Überwachung bei adäquater Therapie durch einen Fachkundigen auch mögliche Komplikationen erfolgreich beherrschbar sind.

\section{Literatur}

1. Kleine-Tebbe J, Fuchs T, Klimek L et al. Die spezifische Immuntherapie (Hyposensibilisierung) mit Allergenen. Positionspapier der Deutschen Gesellschaft für Allergologie und klinische Immunologie, inhaltlich abgestimmt mit dem Ärzteverband Deutscher Allergologen. Allergo J 2000; 9: 317-24.

2. Hepner MJ, Ownby DR, Anderson JA et al. Risk of systemic reactions in patients taking beta-blocker drugs receiving allergen immunotherapy injections. J Allergy Clin Immunol 1990; 86: 407-11.

\section{Dr. Herbert Pötz, Dr. Wolfgang Hemmer, Dr. Stefan Wöhrl, \\ Univ.-Doz. Dr. Reinhart Jarisch, Wien.}

\section{Anmerkung der Herausgeber}

Dieser Leserbrief lädt zur Diskussion ein! Dabei sollten die Beta-Blocker, die allgemein als zumindest relative Kontraindikation einer Hyposensibilisierung gelten (siehe auch J Allergy Clin Immunol 1989; 84: 129-30 und Allergy 1998; 53 (Suppl 44): 1-42) und die nur in Deutschland so gesehenen ACEHemmer unterschiedlich bewertet werden.

Die Herausgeber

Die Rubrik „Leserbriefe“ dient als Diskussionsforum. Die veröffentlichten Beiträge spiegeln nicht unbedingt die Ansicht der Herausgeber und der Redaktion wider. 